(Physiologisches Laboratorium in Bonn.)

\title{
Ob die Totalexstirpation des Pankreas mit Nothwendigkeit Diabetes bedingt.
}

\author{
Von
}

\section{Eduard Pflüger.}

Wenn man die Literatur des experimentellen Diabetes durchmustert, findet man öfter die Behauptung, dass die Totalexstirpation des Pankreas keineswegs immer von Diabetes gefolgt sei. Gerade in neuester Zeit wurden von H. Lüthje ${ }^{1}$ ) solche als "Totalexstirpationen" bezeichnete Fälle beschrieben, bei denen wie bei der partiellen Abtragung des Pankreas nur vorübergehende Glykosurien auftraten. Da diese Form der vorübergehenden Glykosurien, wie allgemein zugegeben wird, wenigstens theilweise auf nervöser Basis steht, und da nach der Totalexstirpation bis zum Tode des Thieres stets Reizzustände in der Abdominalhöhle vorhanden sind, muss untersucht werden, ob es wohl möglich ist, die Totalexstirpation so schonend auszuführen, dass auch sie keine dauernde Glykosurie erzengt. Die merkwürdigen, von $H$. Lüthje gemeldeten vorübergehenden Glykosurien verdienten auch desshalb besondere Beachtung, weil ein ausgezeichneter Professor der Chirurgie, Herr Küttner in Tübingen, die Operation ausgeführt hatte.

Die Frage ist aber von zu grundsätzlicher Wichtigkeit, als dass ich es nicht für nothwendig erachtet hätte, mir durch eigene Untersuchung ein Urtheil zu bilden. Begünstigt war ich dadurch, dass mir einer der grössten jetzt lebenden Meister der Chirurgie, mein College Oscar Witzel, zur Seite stand und die Totalexstirpationen selbst vollzog, während ich den physiologischen Theil der Arbeit übernahm. Die Ergebnisse, zu welchen ich gelangte, haben mir die Ueberzeugung versehafft, dass vor 0 . Witzel nur ausnahmsweise wirkliche Totalexstirpationen des Pankreas ausgeführt worden sind. Ich bin desshalb Herrn Collegen O. Witzel zu besonderem Dank verpflichtet, dass er in der vorhergehenden Abhandlung seine Operationsmethode genauer beschrieben hat.

1) Hugo Lüthje, Deutsches Arch. f. klin. Medicin Bd. 79 S. 498.1904 und ebendaselbst Bd. 80 S. 98.1904. 
Wir verzeichnen 13 Totalexstirpationen des Pankreas beim Hunde mit dem Ergebniss, dass stets Diabetes auftritt, der trotz absoluter Nahrungsentziehung bis zum Tode des Thieres, ohne jemals auszusetzen, anhält. In einen Falle überlebte der Hund die Totalexstirpation 16 Tage, in einem anderen Falle 19 Tage. In jenem am genauesten untersuchten Falle bat Herr Professor M oritz Nussb a um die Güte gehabt, nach dem Tode die Section auszuführen und dann durch Serienschnitte des Duodenums und des Mesenteriums die absolute Abwesenheit jeder Pankreaszelle festzustellen. College $\mathrm{Nussbaum}$ hat mir die ausgezeichnet schönen von ihm gefertigten Präparate zur Prüfung vorgelegt. Es ist das desshalb sehr wichtig, weil allgemein angenommen wird, dass eine absolut vollständige Exstirpation des Pankreas unmöglich sei. So spricht sich auch Thiroloix ${ }^{1}$ ) aus, einer der erfabrensten Forscher auf diesem Gebiete. Ehenso E. Hédon ${ }^{2}$ ), ein ausgezeichneter Kenner des Diabetes, der Hunderte von Exstirpationen selbst ausgeführt hat.

Man sollte ja meinen, dass mikroskopische Reste von Pankreasgewebe, welche der Totalexstirpation entgehen, keine praktische Bedeutung haben können. Wenn ich aber das Verhalten der von o. Witzel operirten Hunde mit den gewöhnlichen Schilderungen vergleiche, muss ich schliessen, dass jene Reste nicht vollständig exstirpirten Pankreasgewebes sich noch sehr entschieden bemerkbar machen. Wie schon Wilhelm Sandmeyer ${ }^{3}$ ) richtig hervorhebt, fehlen nach Totalexstirpation - was erstaunlich wichtig ist Polydypsie, Polyphagie, Polyurie oder sind in nur geringem Maasse vorhanden. Sie sind, wie Sandmeyer ausdrücklich betont, das Zeichen der partiellen Exstirpation.

Nicht jede partielle Exstirpation erzeugt den unstillbaren Durst und Hunger mit riesiger Harnabsonderung. Das Wesentliche liegt in der Art des operativen Eingriffes und der Lage der nicht vollständig exstirpirten Reste. Ebenso fehlt nach wirklicher Totalexstirpation das zeitweilige Aussetzen der Glykosurie gänzlich. In unseren Versuchen trat der Diabetes - abgesehen von einem Falle - immer in den ersten 24 Stunden ein und dauerte, allmählich an Stärke abnehmend, bis zum Tode. Der in der Blase des Cadavers vorhandene Harn enthielt noch einige Zehntelprocent Zucker.

Ein Ausnahmefall betrifft eine Totalexstirpation des Pankreas,

1) J. Thiroloix, Diabète pancréatique p. 95. 1892.

2) E. Hédon, Travaux de Physiologie 1898 p. 39, 71, 74.

3) W. Sandm eyer, Zeitsehr. f. Biol. Bd. 29 S. 94.1892. 
welche bei einem Hunde von $15,5 \mathrm{~kg}$ wegen besonderer Länge des Pankreas, die $49 \mathrm{~cm}$ betrug, ungewöhnlich lange Zeit in Anspruch nahm. Der Hund überlebte die Operation nur $2^{1 / 2}$ Tage und hatte nie eine Spur Zucker im Harne. Wäre der Hund länger am Leben geblieben, würde die Glykosurie noch sicher eingetreten sein. Denn auch andere Autoren haben solche, ja grössere Verspätung für das Eintreten der Glykosurie beobachtet. W. Sandmeyer ${ }^{1}$ ) berichtet, dass der Eintritt sich bis zu 68 Stunden, Thiroloix²), dass er sich bis zum siebenten Tag nach der Operation verzögert habe. Es ist wohl denkbar, dass die Leber, welche bei einer lange dauernden Operation sich abkuhlt und auf andere Weise geschädigt wird, eine Art Paralyse erleidet, so dass sie die Zuckererzeugung nicht zu leisten vermag bezw. zur Erholung längere Zeit beansprucht.

Der Widerspruch, den unsere Ergebnisse gegenüber den von H. Lüthje veröffentlichten darbieten, veranlassle uns, sowohl diesen Forscher wie Herrn Prof. Küttner um nähere, Auskunft zu bitten. Beide Herren waren so gütig, uns mitzutheilen, dass der Ausdruck "Totalexstirpation“ nicht strictissimo sensu zu nehmen sei. Denn Serienschnitte hätten ergeben, dass am Duodenum Reste von Pankreasgewebe nachgewiesen werden konnten. Die merkwürdige Thatsache, dass solche, oft sehr kleine Reste noch eine erhebliche Bedeutung haben, soll in einer späteren Mittheilung von mir eingehender behandelt worden. Bei E. Hédon ${ }^{3}$ ) und J. Thiroloix ${ }^{4}$ ) findet man viele thatsächliche Belege für diese Paradoxie.

Man könnte nun endlich noch den Einwand erheben, dass bei unseren Versuchen nicht das Fehlen des Pankreas, sondern die bis zum Tode wund bleibende Bauchhöhle den Diabetes bedinge. Dies wird widerlegt durch die Wirksamkeit kleiner Pankreasreste, welche der Exstirpation entgangen sind, und vor Allem durch die Wochen, ja, Monate lang nach partieller Pankreasexstirpation fehlende Glykosurie, welche später, wie W. Sandmeyer entdeckte, als echter Diabetes doch eintritt, sobald der zurückgebliebene Pankreasrest degenerirt oder resorbirt ist. Ich habe auch zwei solche Fälle untersucht und kann Sandmeyer's Angaben durchaus bestätigen.

1) W. Sandmeyer, Zeitschr. f. Biol. Bd. 29 S. 92.1892.

2) Thiroloix, Diabète pancréatique p. 77. 1892.

3) E. Hédon, Travaux de Physiologie 1898 p. 34, 39, 41, 74.

4) J. Thiroloix p. 30, 95. 
Eduard Pflüger:

Hund 14. 10,2 kg Anfangsgewicht. Totalexstirpation des

\begin{tabular}{|c|c|c|c|c|c|c|}
\hline \multirow{2}{*}{$\begin{array}{r}\text { Datun } \\
1904\end{array}$} & \multirow{2}{*}{$\begin{array}{l}\text { Gewicht } \\
\text { des } \\
\text { Hundes } \\
\text { in } g\end{array}$} & \multirow{2}{*}{$\begin{array}{l}\text { Volum } \\
\text { des } \\
\text { Harns } \\
\text { in } \mathrm{ccm}\end{array}$} & \multirow{2}{*}{$\begin{array}{l}\text { Spec. } \\
\text { Gewicht } \\
\text { des } \\
\text { Harns }\end{array}$} & \multicolumn{2}{|c|}{$\begin{array}{c}\text { PolarimetrischeDrehung } \\
\text { in Grad }\end{array}$} & \multirow{2}{*}{$\begin{array}{c}\text { Zucker- } \\
\text { procente } \\
\text { nach } \\
\text { Fehling }\end{array}$} \\
\hline & & & & $\begin{array}{l}\text { vor } \\
\text { Gährung }\end{array}$ & $\begin{array}{c}\text { nach. } \\
\text { Gährung }\end{array}$ & \\
\hline 10. No & 10200 & $\begin{array}{l}540 \\
\mathbf{3 9 0}\end{array}$ & 1026 & $\begin{array}{l}+3,4 \\
+5,1\end{array}$ & & $+3,7$ \\
\hline 11. & & $\begin{array}{l}550 \\
\mathbf{4 0 0}\end{array}$ & 1033 & $\begin{array}{l}+3,25 \\
+4,4\end{array}$ & & \\
\hline 12. & & $\begin{array}{l}480 \\
330\end{array}$ & 1029 & $\begin{array}{l}+2,56 \\
+2,76 \\
+4,0\end{array}$ & $-0,20$ & $+3,1$ \\
\hline 13. & & $\begin{array}{l}470 \\
\mathbf{3 2 0}\end{array}$ & 1030 & $\begin{array}{l}+2,36 \\
+\mathbf{3 , 5}\end{array}$ & & \\
\hline 14. & & $\begin{array}{l}400 \\
\mathbf{2 5 0}\end{array}$ & 1024 & $\begin{array}{l}+2,34 \\
+3,7\end{array}$ & & \\
\hline 15: , & & $\begin{array}{l}435 \\
\mathbf{2 8 5}\end{array}$ & 1026 & $\begin{array}{l}+2,23 \\
+3,4\end{array}$ & & $+2,71$ \\
\hline 16. & & $\begin{array}{l}360 \\
\mathbf{2 1 0}\end{array}$ & 1024 & $\begin{array}{l}+2,17 \\
+3,7\end{array}$ & & \\
\hline 17. & & $\begin{array}{l}380 \\
\mathbf{2 3 0}\end{array}$ & 1027 & $\begin{array}{l}+2,54 \\
+4,2\end{array}$ & & $+2,56$ \\
\hline 18. & & $\begin{array}{l}450 \\
\mathbf{3 0 0}\end{array}$ & 1024 & $\begin{array}{l}+2,01 \\
+3,0\end{array}$ & & \\
\hline 19. , & 7600 & $\begin{array}{l}380 \\
\mathbf{2 3 0}\end{array}$ & 1033 & $\begin{array}{l}+2,80 \\
+4,6\end{array}$ & & $+3,29$ \\
\hline 20. & & $\begin{array}{l}480 \\
\mathbf{3 3 0}\end{array}$ & 1025 & $\begin{array}{l}+2,13 \\
+2,47 \\
+\mathbf{3 , 6}\end{array}$ & $-0,34$ & $+2,50$ \\
\hline 21. & & $\begin{array}{l}500 \\
\mathbf{3 5 0}\end{array}$ & 1024 & $\begin{array}{l}+2,10 \\
+\mathbf{3 , 0}\end{array}$ & & $+2,47$ \\
\hline 22. & & $\begin{array}{l}480 \\
\mathbf{3 3 0}\end{array}$ & 1017 & $\begin{array}{l}+1,38 \\
+1,48 \\
+\mathbf{2 , 1}\end{array}$ & $-0,10$ & $+1,55$ \\
\hline 23. & & $\begin{array}{l}410 \\
\mathbf{2 6 0}\end{array}$ & 1021 & $\begin{array}{l}+1,70 \\
+2, \%\end{array}$ & & $+2,01$ \\
\hline 24. & & $\begin{array}{l}450 \\
300\end{array}$ & 1016 & $\begin{array}{l}+1,24 \\
+1,69 \\
+\mathbf{2 , 5}\end{array}$ & $-0,45$ & $+1,68$ \\
\hline 25. & 6330 & $\begin{array}{l}430 \\
\mathbf{2 8 0}\end{array}$ & $1022^{\circ}$ & $\begin{array}{l}+2,50 \\
+3,8\end{array}$ & & $+2,88$ \\
\hline 26. & Tod & $\begin{array}{l}275 \\
125\end{array}$ & 1009 & $\begin{array}{l}+0,50 \\
+1,1\end{array}$ & & \\
\hline
\end{tabular}


Ob die Totalexstirpation des Pankreas mit Nothwendigkeit Diabetes bedingt. 185

Pankreas durch Prof. Oscar Witzel am 9. November 1904.

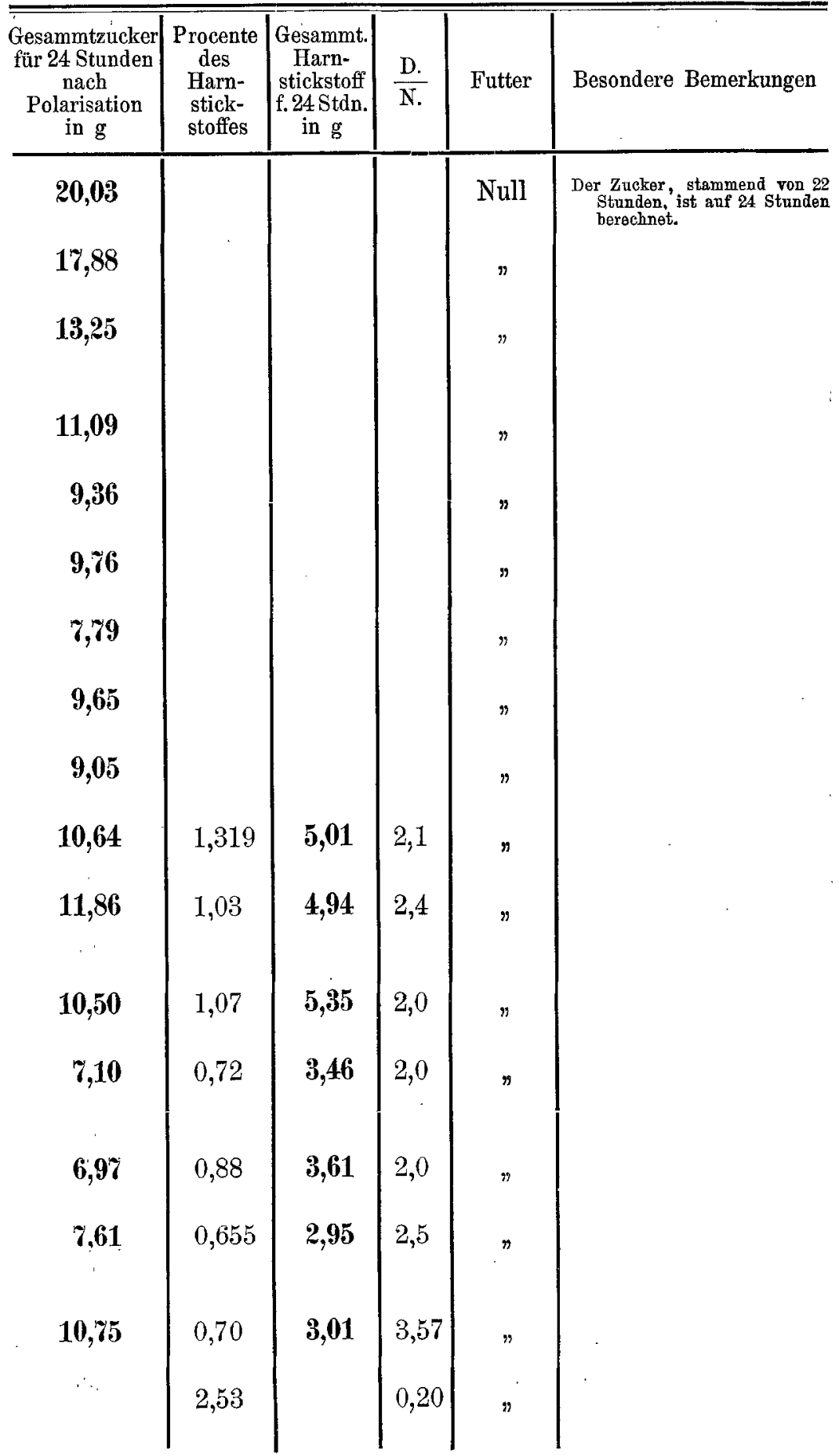


Ich lasse nun als besonders lehrreiche Beispiele einige Uebersichten folgen, welche bezeugen, wie in allen Fällen nach einer wirklichen Totalexstirpation sich grundsätzlich der Verlauf des Diabetes gestaltet hat. Die hier mitzutheilenden Beispiele betreffen die beiden Hunde, welche die Totalexstirpation des Pankreas 16 und 19 Tage überlebten. Stets ist nach eingetretenem Tode des Thieres die absolute Vollkommenheit der Totalexstirpation durch genaueste Untersuchung sicher gestellt sowie als Todesursache der eiternde Abscess erkannt worden. Nicht das Fehlen des Pankreas, nicht der Diabetes sind die Ursache der kurzen Lebensdauer der Thiere, sondern die durch den Zuckergehalt der Säfte verhinderte Heilung der eiternden Wunden. Der Sandmeyer'sche Diabetes beweist dies auch mit hinreichender Sicherheit.

(Siehe die Tabellen auf Seite 184 und 185.)

Der Harn des Hundes reagirte ausnahmslos sauer. In Stab 3 bedeutet die fette Zahl das wirkliche Volum, die darüber stehende, um wieviel durch das Spülwasser des Käfigs das Volum vergrölsert ist. - In Stab 5 bedeutet die fette Zahl den wirklichen Zuckergehalt des Harnes. Von den zwei anderen, darüberstehenden Zahlen bedeutet die obere die im Rohr von $189,7 \mathrm{~mm}$ Länge vor der Gährung beobachtete Drehung des verdünnten Harnes. Die darunterstehende Zahl gibt die nach der Gährung corrigirte Drehung an. Sie ist der Rechnung zu Grunde gelegt, weil ja die Titration nicht blos durch den Zucker, sondern auch noch durch andere Stoffe beeinflusst ist, die keine Zucker sind. Die Section des Hundes ist von dem Professor der Anatomie Hern Dr. Moritz Nussbaum ausgeführt. Als Todesursache haben sich ergeben mehrfache Perforationen des Duodenums und Geschwüre der Schleimhaut in nächster Nachbarschaft der Durchbohrungen.

Die merkwürdigste Thatsache ergab die Leber, weil sie ohne Gallenblase $590 \mathrm{~g}$ wog, also $8,37 \%$ des Körpergewichts. Solches ungeheure Gewicht kommt sonst nur bei Kohlehydratmästung vor. Hier lag aber eine Nahrungsentziehung von fast drei Wochen vor, und der Hund hatte in 16 Tagen $35 \%$ an seinem Gewicht verloren, während die Leber zugenommen zu haben scheint. Denn bezieht man das bei der Section gefundene Lebergewicht von $530 \mathrm{~g}$ auf das Anfangsgewicht des Thieres von $10200 \mathrm{~g}$, so macht die 
Leber immer noch 5,2\% des Körpers aus, was ausserordentlich hoch ist, und was um so mehr auffällt, weil der Hund ungefähr eine Woche vor der Operation keine Nahrung mehr erhalten hatte. Die Leber war stark gelb marmorirt. Der Gehalt an Glykogen war gleich Null; aber der Gehalt an Fett betrug $47,5 \%$, so dass, weil die gesammte Trockensubstanz 58,5\% ausmachte, auf Stickstoffsubstanzen und Salze nur $11 \%$ kommen. - Beim Pankreasdiabetes findet nach schnellem Schwund des Glykogenes ein reichlicher Ersatz desselben durch Einwanderung von Fett in die Muskeln, besonders aber die Leber statt. Bei hinreichend langer Lebensdauer, wie sie bei dem Sa n d m e y er'schen Diabetes vorkommt, schwindet schliesslich fast alles Fett aus dem Körper, der nur noch aus Knochen, Muskeln, Nerven und Drüsen besteht. In der Leber hat die Eiweisssubstanz zugenommen, trotz der allgemeinen Abmagerung; das Fett ist bis auf 2 bis $3 \%$ aus ihr geschwunden. Bei dem Sandmeyer'schen Diabetes fängt die Zuckerausscheidung stark abzunehmen an, wenn das Fett fast vollkommen aufgebraucht ist, obwohl die reichlichste Eiweissnahrung zugeführt wird. Das spricht sehr stark für das Fett als Quelle des Zuckers. Genaueres in einer späteren Arbeit.

\section{Hund 12.}

Gewicht $12 \mathrm{~kg}$. - Prof. O. Witzel macht die Totalexstirpation des Pankreas am 24. September 1904. Der Hund erhält keine Nahrung. Ueberlebt die Operation $18 \frac{1}{2}$ Tage.

\begin{tabular}{|c|c|c|c|}
\hline $\begin{array}{c}\text { D a t u m } \\
1904\end{array}$ & $\begin{array}{l}\text { Procent- } \\
\text { gehalt an } \\
\text { Zucker }\end{array}$ & $\begin{array}{c}\text { Zuckermenge } \\
\text { von } 24 \text { Stunden } \\
\text { in } \mathrm{g}\end{array}$ & $\begin{array}{c}\text { Volum des } \\
\text { Harns } \\
\text { in ccm }\end{array}$ \\
\hline 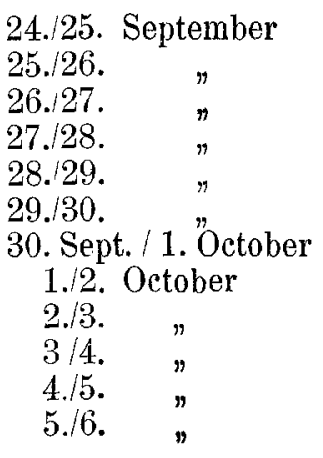 & $\begin{array}{l}1,9 \\
2,4 \\
4,6 \\
5,5 \\
4,05 \\
3,7 \\
5,2 \\
4,6 \\
5,6 \\
5,3 \\
3,0 \\
2,9\end{array}$ & $\begin{array}{r}14,19 \\
11,98 \\
36,66 \\
26,65 \\
26,33 \\
30,36 \\
15,05 \\
21,35 \\
12,56 \\
8,24 \\
6,06 \\
3,63\end{array}$ & $\begin{array}{l}750 \\
462 \\
790 \\
480 \\
500 \\
820 \\
290 \\
460 \\
225 \\
155 \\
200 \\
125\end{array}$ \\
\hline
\end{tabular}


188 Eduard Pflüger: Ob die Totalexstirpation des Pankreas etc.

\begin{tabular}{|c|c|c|c|}
\hline $\begin{array}{c}\text { D a } \mathrm{t} \mathrm{u} \mathrm{m} \\
1904\end{array}$ & $\begin{array}{l}\text { Procent- } \\
\text { gehalt an } \\
\text { Zucker }\end{array}$ & $\begin{array}{c}\text { Zuckermenge } \\
\text { von } 24 \text { Stunden } \\
\text { in } \mathrm{g}\end{array}$ & $\begin{array}{c}\text { Volum des } \\
\text { Harns } \\
\text { in ccm }\end{array}$ \\
\hline 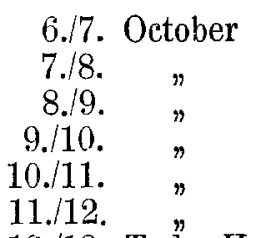 & $\begin{array}{l}1,3 \\
1,0 \\
0,8 \\
1,1 \\
4,9 \\
2,2\end{array}$ & $\begin{array}{l}2,55 \\
1,88 \\
0,98 \\
2,01 \\
5,13 \\
3,36\end{array}$ & $\begin{array}{l}190 \\
180 \\
130 \\
185 \\
105 \\
155\end{array}$ \\
\hline
\end{tabular}

12.13. Tod. Harn in der Blase enthält Zucker. Section: Eiteriger Abscess.

Es ist mir endlich eine angenehme Pflicht, den herzlichsten Dank auszusprechen für die mir gewährte werthvolle Unterstützung. meinem hochverehrten Collegen Herrn Professor O. Witzel sowie dessen Assistenten, besonders Herrn Oberarzt Dr. Friedrich Wenzel, und endlich dem chemischen Assistenten des physiologischen Institutes, Herrn Dr. Kurt Moeckel, welcher die Stickstoffbestimmungen nach $\mathrm{Kjeldahl}$ für mich ausgeführt hat. 